\title{
Convivencia Escolar para el aprendizaje y buen trato de todos: Hacia una mejor comprensión del concepto
}

\section{School Coexistence for learning and good treatment of all. Towards a better understanding of the concept}

DOI: http://dx.doi.org/10.17981/cultedusoc.8.2.2017.01

María Isidora Mena Edwards y Marcial Ramón Huneeus Villalobos ${ }^{2}$

\begin{abstract}
Resumen
Como todos los conceptos que la política pública pone en boga, la "Convivencia Escolar" es hoy en día un constructo polisémico, que muchos cuando se les pide definir o explicar qué es lo que permite una "buena" convivencia escolar, deben reconocen desconocimiento. La experiencia en terreno muestra tendencias: quienes asocian convivencia escolar a un ambiente cálido, de relaciones humanas amables y no violentas; y otros que asocian buena convivencia a un ambiente disciplinado entendiendo por tal ordenado, respetuoso, obediente al quehacer escolar. Valoras UC, un programa de "Convivencia escolar para el aprendizaje y buen trato de todos" de la P. Universidad Católica de Chile, propone que la convivencia escolar debe adjetivarse por el objeto de la escuela, el aprendizaje integral de todos. La propuesta consiste en "pensar la escuela como una comunidad de aprendizaje con la meta del aprendizaje y buen trato de todos" (Valoras UC, 2017). El aprendizaje requiere como contexto social buen trato entre compañeros y un vínculo afectuoso con los docentes, ambos son fundantes de la confianza y la disposición fisiológica para que se activen las redes neuronales relacionadas con un aprendizaje significativo y comprensivo. Esto significa que si la convivencia que hay en la escuela no permite que todos aprendan y que esto sea en un ambiente de buen trato, no estaríamos hablando de la "buena convivencia escolar". La Buena Convivencia Escolar debiera expresarse en un buen clima, que permite buenos aprendizajes, de todos.
\end{abstract}

Palabras Clave: Convivencia escolar, aprendizaje, Programa Valoras UC.

Recibido: Agosto 10 de 2017 / Aceptado: Septiembre 8 de 2017

\footnotetext{
${ }^{1}$ Pontificia Universidad Católica de Chile. Directora ejecutiva Valoras UC. Correo: imenae@uc.cl

${ }^{2}$ Fundación Patio Vivo de Chile. Coordinador área Educación. Correo: mrhuneeus@gmail.com
} 


\begin{abstract}
Like all the concepts that public policy puts in vogue, the "School Coexistence" is nowadays a polysemic construct, which many when asked to define or explain what it is that allows a "good" school coexistence, must recognize ignorance. The experience in the field shows trends: those who associate school coexistence with a warm environment, with friendly and non-violent human relations; and others that associate good coexistence with a disciplined environment, understood as being orderly, respectful, obedient to school work. Valoras UC is a program of "School Coexistence for the Learning and Good Treatment of All" of the P. Universidad Católica de Chile, proposes that school coexistence should be adjectived by the object of the school, the integral learning of all. The proposal consists of "thinking of the school as a learning community with the goal of learning and good treatment for all" (Valoras UC, 2017). The learning requires a good social relationship between peers and an affectionate bond with the teachers, both are foundational of the trust and the physiological disposition so that the neural networks related to a meaningful and comprehensive learning are activated. This means that if the coexistence in the school does not allow everyone to learn and that this be in a good treatment environment, we would not be talking about "good school life". The Good School Coexistence should be expressed in a good climate, which allows good learning, of all.
\end{abstract}

Keywords: School life, learning, Valoras UC Program.

\title{
Introducción
}

En Chile, a partir del Decreto Supremo de Educación N ${ }^{0}$ 381, 2013, se agregó a las pruebas nacionales de rendimiento académico un cuestionario que mide los llamados Otros Indicadores de la Calidad Educativa, posteriormente denominados Indicadores de Desarrollo Personal y Social. Esto implica que la calidad de las escuelas es demostrada a través de indicadores de rendimiento académico y en un 33\% por "otros indicadores de calidad", entre ellos clima de convivencia escolar. Desde la perspectiva de convivencia para el aprendizaje y buen trato de todos, una escuela de calidad debiera tener ambos puntajes altos.

En el camino a apoyar a escuelas para una mejor convivencia que permita el aprendizaje y buen trato de todos, el trabajo que se presenta se propuso explorar y sintetizar estudios y reflexiones de destacados autores ocupados de aquella convivencia escolar que permite aprender a ser personas integrales e íntegras. ¿Qué distinciones aportan a este constructo de convivencia escolar?.

A principios del siglo XX, los historiadores españoles usaron el término "convivencia" para describir épocas de la historia en que hubo relaciones pacíficas entre pueblos usualmente en guerra. Casi cien años después, en ramos de educación, académicos de España y Latinoamérica retoman el concepto de "convivencia" para referirse a uno de los ejes de la calidad educativa propuestos por la UNESCO (Delors, 1996), que habla de aprender a "vivir juntos, aprender a vivir con los demás". La calidad educativa debió incorporar la capacidad de la escuela para lograr interacciones pacíficas, en una época que aumenta fuertemente la diversidad por efecto de la cobertura escolar, la integración de estudiantes migrantes y las políticas de inclusión de estudiantes con necesidades educativas diferentes. "Convivencia" se significó como un ideal de 
prácticas respetuosas y tolerante con las diferencias, valoración de la interdependencia, pluralidad, enfrentamiento pacífico de los conflictos, participación democrática, entendimiento mutuo. (Esperanza, 2001; Gallardo, 2009; Touriñán, 2005).

En la tradición inglesa, por su parte, si bien se sigue usando el concepto de "convivencia" en el campo de la historiografía, en educación se habla de "coexistencia", que se diferenciaría de "convivencia" tal como se acuña en Iberoamérica en que este último más que enfatizar el concepto de coexistencia, refiere a la interacción positiva y proactiva en las distintas áreas de la vida en común de la escuela (Gallardo, 2009; Ray, 2005). Carbajal (2013) propone mantener el término convivencia en su forma original, referido a una acción conjunta.

Bazdrech (2009), manteniendo un concepto proactivo de convivencia, define el concepto de una manera que parece útil porque lo "neutraliza" en su núcleo conceptual desmarcándolo de ideales de convivencia, a la vez que aclara que el concepto está afectado por los valores que se quieran sustentar. Neutralizar parece necesario para evitar confusiones y dejar en claro que aún una "mala" convivencia es convivencia y educa. Aclarar su raigambre valórica por su parte es, además de necesario, útil operacionalmente en tanto la formación moral y ética es una de las vertientes formativas con más sustento teórico y empírico en el ámbito de la convivencia. ${ }^{3}$

Bazdrech (op cit) propone que "la convivencia está formada por una trama de relaciones personales y sociales de los actores educativos que constituyen la cotidianeidad de la escuela. Las relaciones personales y sociales concretas y observables, que constituyen la convivencia escolar provienen, al menos parcialmente, de los valores que se propone "formar" la escuela" (2009: 62). Hirmas y Eroles (2008, UNESCO), describen esta trama como un proceso constructivo permanente de transacciones, negociación de significados, elaboración de soluciones, proceso que va creando un referente común e histórico, que genera un sentido de familiaridad e identidad grupal (pp 18-19). Esta familiaridad y cotidianeidad explica cómo es que la convivencia presenta la paradoja de ser omnipresente al mismo tiempo que invisibilizada.

Fierro (2013) explica que en la convivencia escolar se puede distinguir la dimensión de las interacciones de las personas, y otra, que enmarca a la primera, referida a las políticas y prácticas institucionales. La convivencia entonces se desarrolla en lo que llama "el gran continente de la gestión escolar", que incluye las prácticas normativas y disciplinarias, las de cuidado y aprecio, y las prácticas pedagógicas. Es esta la dimensión que Fierro (op cit) propone como distintiva del concepto de "convivencia escolar".

Entendida así la convivencia, y en tanto constituye una construcción colectiva cotidiana (para bien o para mal), y en tanto es de responsabilidad de todos, tiene gran impacto en el aprendizaje y desarrollo de las personas, sus identidades, sentido de pertenencia, su formación socioafectiva y ética.

3 Entre los autores que aportan propuestas formativas de valores (o moral) está Kohlberg (1997) y su propuesta del desarrollo del Juicio Moral; Durkheim (2000) y sus propuestas de socialización; Lev Vygostky (1993) desde el estudio del desarrollo cognoscitivo han inspirado propuestas de formación en valores. Desde la filosofía política y del derecho han derivado programas formativos de valores desde los derechos humanos universales, tales como la educación en la cultura de paz, educación de ciudadanos, y de resolución pacífica de conflictos (Martínez de M., 1999). 
Cabe aclarar que los pocos autores que han trabajado en el concepto de convivencia coinciden en que aún cuando ha habido avances, este es un concepto amplio, complejo y dinámico, al que le falta aún un corpus teórico bien establecido (Chaparro y colaboradoras, 2015). Su abordaje práctico es también complejo, y tal como había sugerido Fierro en el 2013, la convivencia en una institución se debiera abordar siempre de manera provisional y fragmentaria, por aproximaciones sucesivas.

\section{Visibilizar y actuar en la convivencia escolar}

Un primer paso para moverse del nivel conceptual a la cotidianeidad de la convivencia en la escuela es, en primer lugar, aclarar el status (su relevancia) y la orientación valórica que se le desea otorgar; en segundo lugar, visualizar el enfoque teórico de las intervenciones y finalmente las intervenciones propiamente tal. Cabe reconocer, antes de hacer el recorrido que va desde las conceptualizaciones a las propuestas concretas, que uno de los factores que dificulta sistematizar un estado del arte, es que suele haber saltos entre las conceptualizaciones de convivencia y las bajadas a la cotidianeidad escolar. Las primeras, cuando se hacen, suelen tener enfoques analisticos, complejas y relacionarse a la trama de interacciones escolares que a la vez constituyen que forman en convivencia, y en cambio las estrategias concretas de bajada suelen referir a acciones muy restringidas y nada de transformadoras del mismo entramado.

El foco de la política pública general dominante en la mayoría de los países de LA no ha estado centrado en un cambio en la trama de relaciones personales y sociales de los actores educativos, sino más bien en currículum, evaluación e integración. El entramado convivencial no ha sido visibilizado en su relación con el aprendizaje. Los temas de convivencia aparecen ligados más bien a los problemas que está teniendo la operativa del sistema escolar tradicional en el contexto de demandas siglo XXI: violencia, indisciplina y dificultad en la inclusión de "otros" diferentes. Esta aclaración se requiere hacer para que el lector no crea que es él quien pierde a veces el hilo conductor de las propuestas: son las mismas propuestas que aún están en una fuerte tensión entre las definiciones conceptuales, las convicciones más profundas de los propios autores de políticas o programas, la realidad de la política educacional concreta y los problemas de las escuelas.

Respecto de la invisibilizada convivencia, la UNESCO ha impactado en Iberoamérica declarando la convivencia como un pilar básico de la calidad educativa, asunto que queda acuñado a través de Delors et al. (1996), Las políticas educativas proponen la convivencia como eje en sus planes en toda LA, ateniéndose además a cartas como la de los DDHH y a las respectivas constituciones y sus artículos referidos a calidad educativa y formación integral.

Desde el enfoque de Derechos Humanos, hay tres dimensiones relacionadas a la convivencia que dan origen a distintos focos de intervención en la escuela: inclusión, democracia y paz. Hirmas y Carranza (2009), y Unesco, describen estas tres dimensiones de la siguiente manera: 


\section{Convivencia inclusiva}

La inclusión alude a la experiencia de pertenecer y a la de ser reconocido y valorado en la propia identidad. Promover inclusión significa promover comunicación, diálogo y colaboración para trabajar hacia el bien común. Incluye la organización institucional con políticas que aseguren permanencia de todos los estudiantes.

\section{Convivencia democrática}

La convivencia democrática tiene que ver con la experiencia de participar en la vida compartida, aprendiendo herramientas socioemocionales, reflexivas, para trabajar con otros, resolver los conflictos, establecer los acuerdos que regulen la vida en común, y aprendiendo a valorar la solidaridad. Aspectos de la vida escolar propios de una vida democrática son las normas y reglamentos en función de valores, construidos participativamente, aplicados en forma consistente, justa y con sanciones con carácter formativo; participación de las familias; manejo formativo de conflictos; diálogo moral y académico como estrategia pedagógica en las diferentes asignaturas; e instancias de representación de los distintos sectores de la escuela, a través de mecanismos participativos y equitativos.

\section{Convivencia pacífica}

Convivencia inclusiva y democrática construyen las bases para que haya paz. Convivir en paz refiere a capacidades personales para interactuar con respeto por los otros, cuidado del bien común, confianza en otros y en la institución, así como a características institucionales, tales como la capacidad de actuar preventivamente, atender a conductas de riesgo, un enfoque de reparación de daño y reinserción.

Las políticas públicas que abordan convivencia en efecto han estado ya sea secuencial o paralelamente abordando estas tres dimensiones. Se puede evaluar su fortaleza según afectan la estructura del sistema, la evaluación y supervisión, el financiamiento, y la capacitación.

\section{Respecto de los enfoques de formación de la convivencia}

Bazdrech (2009) explica que la formación, entendida como el acompañamiento de los estudiantes para forjar sus formas de ser y convivir en el mundo, ha tenido en la historia de la educación fundamentalmente dos enfoques educativos.

El primer enfoque, explica, se propone aprovechar la vida cotidiana escolar para formar en las prácticas requeridas para vivir la escuela. Según sean los vínculos de trato y de intercambio entre los actores será la relación pedagógica y la socialización que se entregue, es decir, los aprendizajes sobre los significados y operaciones cotidianas del mundo social, los límites y las posibilidades de actuar. La convivencia misma sería el pedagogo real de constitutivos morales de las relaciones personales y sociales. Los valores identificados en esa trama de la vida escolar indicarán la 
eficacia de la formación en valores propuesta por la escuela, independientemente del proceso pedagógico explícito que la escuela haya seleccionado para la "formación en valores", de sus alumnos.

Carbajal (2013) llama a este enfoque "enfoque curricular progresista", y lo relaciona con las propuestas de Dewey (1967) de construir en las escuelas comunidades democráticas que promuevan el crecimiento humano y las habilidades ciudadanas, así como con el concepto de Freire (1970) de "praxis" como una acción de reflexión educativa que incidirá en la construcción de relaciones sociales más justas. Ambas teorías de socialización tienen en común el fomento de la comunicación abierta a través del diálogo respetuoso, y la deliberación crítica que permite ir transformando los obstáculos que se interponen en la realización personal. Shyman (2011) llama a estos enfoques "educación para una ciudadanía crítica”, que cuestionan las estructuras que producen la injusticia social (ejemplos son Banks, 2008; Fischman y Hass, 2012; Westheimer y Kahne, 2004).

Cabe señalar que mientras el educador americano Dewey (op cit) buscaba mejorar una sociedad ya liberal y democrática, como la de Estados Unidos, el brasileño Freire (op cit) proponía una transformación radical de las relaciones de poder fuertemente autoritarias y desiguales como las que existen en América Latina a unas democráticas. Junto a las propuestas teóricas de Dewey y Freire, Carbajal (2013) propone agregar la vertiente de "la educación para la justicia social", que partiendo de las anteriores, agrega la necesidad de reconocer las diferencias culturales, la fluctuación del estatus social y la intersección en la escuela de múltiples identidades,tanto en estudiantes como en docentes-, instando a buscar equidad y colaboración (Carbajal cita a Aronson, 2001; Cohen, 2006; Morrison et al., 2008).

Fierro y Tapia (2013) proponen para este enfoque que aborda el fenómeno social de la convivencia el concepto de "analítico". La inclusión, exclusión, participación, resolución de conflictos, disciplina o violencia, se juegan en la enseñanza, normas, trato a las diferencias, relación con las familias, interacción profesor-estudiantes. La convivencia democrática o la educación para la justicia social se convierten en el currículum explícito e implícito a través de pedagogías que promueven la resolución pacífica de los conflictos (peacemaking), la equidad, la paz (peacebuilding), y de una distribución más equitativa del poder tal que los estudiantes tengan más oportunidades para ejercitar la ciudadanía activa en las escuelas.

El segundo enfoque formativo otorga tiempo y actividades especiales para educación moral y ética, segmentando por ciclos lo que se quiere enseñar y aprovechando el diseño curricular de las asignaturas para incorporar contenidos y prescripciones necesarias para convivir de una cierta manera. La política educativa de la mayoría de los países latinoamericanos se ha inclinado hacia la forma de asignatura, en un enfoque de "formación cívica y ética" a través de currículum explícitos de las asignaturas, explica Fierrro (op cit), asunto que queda restringido al no estar aparejado con intervenciones en el tejido de las interacciones de la escuela, del vehículo formativo.

A este enfoque Carbajal (2013) le ha llamado "enfoque restringido" de la convivencia, y semejante a las teorías curriculares de eficiencia-social, focaliza el desarrollo de las habilidades individuales y ofrece pocas oportunidades de agencia 
y poder respecto de las interacciones en la escuela misma. Dentro de este enfoque se enmarcan en general las intervenciones de convivencia orientadas a prevenir la violencia. Fierro y Tapia (2013) proponen hablar del enfoque normativo-prescriptivo, que aborda la convivencia para prevenir la violencia. Sobre el abordaje de la violencia y sus enfoques, como propuesta principal de las intervenciones en convivencia, trata el punto siguiente de este documento.

Con relación a los enfoques de abordaje de la convivencia que han predominado se puede identificar que ellos derivan fundamentalmente del desafío de los DDHH y de los problemas del sistema educacional, más que abordajes que cubran el desafío de las necesidades de cambio de enfoque del sistema educacional ya sea por razones de aprendizaje o de formación para el siglo XXI.

El primer problema que se intenta abordar desde la formación para la convivencia es la violencia, fenómeno altamente preocupante en latinoamérica. Paralelamente o en segundo lugar, la disciplina. Furlan y Spitzer (2013) explican que los estudios de violencia y disciplina han aumentado en forma exponencial en las últimas décadas, claro que, como dice Chaparro et al (2015) en ambos casos, se ha tendido a reducir el concepto de convivencia a solo parte de las dimensiones. Chaparro acusa a los enfoques de violencia el que tienden a criminalizar a los jóvenes, y se podría agregar que en el caso de la disciplina la tendencia es a acusar negligencia de las familias. Siguiendo con la participación y la inclusión, que han sido también focos, se puede decir respecto de la primera un esfuerzo en que todos participen en ciertas decisiones de gestión (que podría dudarse que en ellas competa la participación democrática de todos), siendo mucho menor el énfasis en la participación en las clases de los estudiantes, en la participación aportando a la constitución de "comunidades" de curso los apoderados y a la participación en las decisiones disciplinarias, metodológicas y de planificaciones de los aprendizajes esperados de los docentes. Finalmente, los enfoques para abordar la inclusión, que también se ha asociadas a temas de convivencia, en general las políticas públicas enfocan el desafío desde las adaptaciones curriculares y/o metodológicas, en un enfoque individual, estigmatizando y desaprovechando la diversidad para el aprendizaje de todos.

En síntesis, son enfoques en los que los factores institucionales, culturales y estructurales del fenómeno de la convivencia escolar, son generalmente subvalorados a la hora de las propuestas concretas.

\section{Intervenciones en convivencia}

El fenómeno de la Violencia como hecho social preocupa fuertemente en LA y también en el mundo. Las intervenciones en convivencia por tanto terminan apuntando muchas veces directamente a la violencia, para prevenirla o enfrentarla. Ultrech ya en 1997 muestra que la cuestión de la violencia en la escuela ha sido priorizada en Estados Unidos y en la mayor parte de los países europeos. Es tan fuerte el énfasis en violencia que tienen los programas, que conceptualmente se suele contraponer "convivencia" a "violencia", fomentándose la convivencia escolar "como una apuesta política para regular y disminuir el impacto de los conflictos escolares que se mani- 
fiestan a través de la indisciplina y la violencia en las escuelas" (Furlán, Saucedo y Lara, 2004, p. 28). Se asume que la convivencia se logra mediante la "formación en valores", y la violencia es expresión de una "ausencia de valores" en los educandos y de tensiones vividas en una familia y en una sociedad compleja, también violenta y en crisis recurrente (Ianni, 2002).

PREAL (2003) reconoce dos grandes orientaciones en el abordaje de la violencia escolar: Las estrategias restringidas y la de carácter amplio:

Dentro de las estrategias de carácter restringido, están las de "tolerancia cero", "mano dura", así como las dirigidas de modo focalizado a "jóvenes vulnerables" o a "rehabilitar" infractores y pandilleros. Se podría agregar la llamada "seguridad escolar", que consiste en lograr (desde fuera) "el orden" en las escuelas con apoyos que provienen de otras instancias. Existe consenso explica PREAL en reconocer ineficacia en estas estrategias en el mediano y largo plazo, tanto porque son intervenciones desde afuera imponiendo un orden, tanto como porque no abordan las causas profundas que originan dichos fenómenos (referencias a Akiba et al., 2002; Gladden, 2002; PREAL, 2003; Debarbieux, 2003; Bickmore, 2004; Blaya, 2010; IIDH, 2011).

Las estrategias de carácter amplio tienen un carácter preventivo, se dirigen a toda la población escolar, asumiendo la violencia como un problema estructural que incluye a la institución escolar en su totalidad. Sonia Eljach en un estudio que publica la UNICEF (2011) reafirma que hay un complejo cuadro de prácticas violentas en las escuelas acusando una seria crisis en las relaciones entre estamentos de la comunidad educativa. Las estrategias de carácter amplio combinan estrategias para abordar participativa y reflexivamente las violencias institucionales, atención a formas de discriminación, así como en la promoción del trabajo en equipo, comunicación y diálogo, pensamiento crítico, deliberación de casos, reparación de daño; la formación ciudadana, resolución de conflictos, y finalmente, en acciones orientadas a apoyar a aquellos estudiantes que presentan rezago académico o que enfrentan situaciones de riesgo social.

Carbajal (2013) usando el concepto de paz positiva y negativa de Galtung (1990), explica que las estrategias de carácter amplio apuntan a una paz positiva, que busca la justicia social, la equidad y la autorrealización. Un ejemplo es la "convivencia democrática" que pone el acento en desarrollar relaciones democráticas y estructuras de participación como elementos esenciales para la construcción y consolidación de la paz. Las estrategias cuyo objetivo directo es formar a los estudiantes para disminuir actos de violencia entre los alumnos/as, lo que se califica como una concepción restringida de la convivencia, contiene un enfoque de paz al que le llaman "paz negativa”. Esta forma de intervenir reforzaría el statu quo al no confrontar la violencia estructural.

La revisión que hace Gladden el 2002 de las estrategias para reducir la violencia en escuelas, concluye que los elementos centrales de experiencias exitosas son, combinados: fortalecimiento de las relaciones entre estudiantes y entre ellos y docentes; formación de conductas no violentas; una enseñanza efectiva; y la promoción de responsabilidad hacia la cultura y comunidades. La española Rosario Ortega Ruiz y equipo (2011) por su parte, plantean que en el contexto español se demuestra 
que tienen más efectividad los trabajos que asumen una actuación general sobre la convivencia y aceptan que la violencia forma parte de la visión negativa de la convivencia escolar (Díaz Aguado, 1996; Félix et al, 2007; Ortega, Del Rey, y Fernández, 2003; Ramírez y Justicia, 2006; Trianes y Fernández Figarés, 2001; Viguer y Aviá, 2009).

Respecto de las cualidades de las propuestas, Dina Krauskopf en el 2006 para una consultoría pedida por la Organización Panamericana de la salud (OPS) revisó muchas propuestas de abordajes al tema violencia/convivencia realizados en Estados Unidos y diversos países de Europa y América Latina, y seleccionó cincuenta y cuatro de los cuales veinticinco corresponden a América Latina, dieciséis a Europa y trece a EEUU. Destaca que en muchos proyectos (no los seleccionados) no se explicitan los enfoques, seguido de acciones inconsistentes, breves o superficiales (apagar incendios). Otra característica que le llama la atención, también de proyectos no seleccionados, son marcos teóricos avanzados e informados, no acompañados de propuestas operacionales congruentes, o bien que padecen los problemas de brevedad y superficialidad. En los proyectos seleccionados como exitosos, aprecia dos tipos de enmarque: en los hechos que produjeron alarma, y en la violencia en la sociedad misma. Esta última es una tendencia principalmente en América Latina. Las experiencias propiamente tal, tienden a apuntar a tres tipos de objetivos: 1) aminorar el impacto de la violencia proveniente de los factores estructurales, 2) disminuir las formas de solución violenta de los problemas y conflictos y 3) desarrollar una convivencia solidaria y constructiva. Los objetivos se implementan de acuerdo a los enfoques conceptuales de violencia, sociedad y los derechos, así como en relación a los diversos niveles de prevención y atención que se enfaticen. (pp. 86)

En síntesis, el estado del arte de convivencia respecto del enfrentamiento de la violencia ha visibilizado dos enfoques teóricos y de implementación a través de políticas públicas. El primero centrado en disminuir la violencia y el acoso escolar, cuya bajada es a través de acciones reactivas (protocolos, convivencia en tanto asignatura, sistemas de alertas, intervención de profesionales externos a las escuelas, entre otros). Mientras que el segundo enfoque busca producir transformaciones culturales, poniendo su foco en generar nuevas prácticas y dinámicas que reemplacen las situaciones de violencia por una cultura de paz, donde se propician procedimientos democráticos y la convivencia se trabaja de forma transversal tanto implícita como explícitamente. Los antecedentes demuestran que el segundo enfoque ha demostrado ser más eficiente en el mejoramiento de los climas escolares y en la formación en convivencia (Díaz Aguado, 1996; Félix et al, 2007; Ortega, Del Rey, y Fernández, 2003; Ramírez y Justicia, 2006; Trianes y Fernández Figarés, 2001; Viguer y Aviá, 2009).

Ya sea se quiera disminuir la violencia, aumentar los aprendizajes o mejorar la disciplina en la escuela del siglo XXI, pareciera que trabajar para una convivencia escolar centrada en los aprendizajes y en el buen trato es una de las metas prioritarias. Para ello, la gestión de transformaciones culturales es una práctica imprescindible, que debemos seguir intentando comprender a cabalidad de modo de desarrollar estrategias que promuevan la convivencia y el aprendizaje. 


\section{Referencias}

Akiba, M., Letendre, G. K., Baker, D. P., \& Goesling, B., (2002). Student victimization: National and school systems effects on school violence in 37 nations. American Educational Research Journal, 39(4), 829- 853.

Aronson, E. (2001). Nobody left to hate. Teaching compassion after Columbine. Nueva York: H. Holt.

Banks, J. (2008). Diversity, group identity, and citizenship education in a global age. Educational Researcher 37(3), 129-139.

Bazdresch Parada, M. (2009). La vida cotidiana escolar en la formación valoral: un caso. REICE. Revista Iberoamericana sobre Calidad, Eficacia y Cambio en Educación, 7(2), 50-71.

Bickmore, K. (2004). Discipline for democracy? School districts management of conflict and social exclusion. Theory and Research in Social Education, 32(1), 75-97.

Blaya, C. (2010) Violencia en los centros educativos: Perspectivas europeas. En Alfredo Furlán Violencia en los centros educativos conceptos, diagnósticos e intervenciones. Buenos Aires: Noveduc.

Carbajal, P. (2013). Convivencia democrática en las escuelas. Apuntes para una reconceptualización. Revista Iberoamericana de Evaluación Educativa, 6(2), 13-35.

Chaparro, M. et al (2015). Violencia escolar en América Latina y el Caribe: superficie y fondo. UNICEFF.

Cohen, E. (2006). Equitable classrooms in a changing society. Handbook of the Sociology of Education, 265-283.

Debarbieux, E. (2003). Violencia escolar: un problema mundial. www.unesco.org

Delors, J., et al. 1996. La educación encierra un tesoro. Informe de la Comisión Internacional sobre la educación para el siglo XXI. Paris: UNESCO.

Dewey, J., (1967., Democracia y Educación. Una introducción a la Filosofía de la educación, Buenos Aires, Editorial Losada.

Diaz-Aguado,M.J. (1996). Programas de educación para la tolerancia y prevención de la violencia en los jóvenes. Cuatro volúmenes y dos vídeos. Madrid: Instituto de la Juventud, Ministerio de Trabajo y Asuntos Sociales.

Dina Krauskopf (2006). Estado del arte de las experiencias y proyectos de prevencion de la violencia en ambitos escolares, Organizacion Panamericana de la Salud (OPS).

Durkheim, E. (2000), Determinación del hecho moral. Sociología y filosofía, Miño y Dávila Editores, Buenos Aires.

Eljach, S. (2006), Violencia escolar en América Latina y el Caribe: Superficie y fondo.

UNICef Oficina Regional para América Latina y el Caribe Edificio 102, Ciudad del Saber, Clayton Panamá, República de Panamá

Esperanza, J. (2001). La convivencia escolar. Un problema actual. Los problemas de la convivencia escolar. Un enfoque práctico. Madrid: Federación de Enseñanza de CC. OO, 22-40. 
Félix, V., Soriano, M., Godoy, C. y Martínez, I. (2007). Prevención de la violencia y promoción de la convivencia escolar en la comunitat valenciana. Aula Abierta, 1, 2(36), 97-110

Fierro Evans, María Cecilia. (2013). Convivencia inclusiva y democrática: Una perspectiva para gestionar la seguridad escolar. Sinéctica, (40), 01-18.

Fierro, Cecilia y Guillermo Tapia (2013), "Hacia un concepto de convivencia escolar", en Alfredo Furlán y Terry Spitzer (coords.) (2013), Convivencia, disciplina y violencia en las escuelas 2002- 2012, México, ANUIES/COMIE, Colección Estados del Conocimiento, pp. 71-86.

Fischman, G. y Haas, E. (2012). Beyond Idealized Citizenship Education: Embodied Cognition, Metaphors, and Democracy. Review of Research in Education, 36(1), 169-196.

Freire, P. (1970). Pedagogy of the oppressed. Nueva York: The Continuum International Publishing Group, Inc.

Furlán, Alfredo y Terry Spitzer (2013). Convivencia, disciplina y violencia en las escuelas 2002-2012, México, ANUIES/COMIE, Colección Estados del Conocimiento.

Gallardo, P. (2009). Educación ciudadana y convivencia democrática. Pedagogía Social. Revista Interuniversitaria, (Marzo), 119-133.

Gladden, M. (2002). Reducing school violence: Strengthening student programs and addressing the role of school organizations. Review of Research in Education, 26, 263-299.

Hirmas, C. y Eroles, D. (coord.) (2008). Convivencia democrática, inclusión y cultura de paz. Lecciones desde la práctica educativa innovadora en América Latina. Santiago de Chile: Oficina Regional de Educación de la UNESCO para América Latina y el Caribe (OREALC/ UNESCO).

Hirmas, C. y Carranza, G. (2009). Matriz de indicadores sobre convivencia democrática y cultura de paz en la escuela. III Jornadas de Cooperación Iberoamericana sobre Educación para la Paz, la Convivencia Democrática y los Derechos Humanos. San José de Costa Rica.

IANNI, O. (2002). Teorías de la globalización. México: Siglo XXI.

Kohlberg, L.; F.C. Power y A. Higgins (1997). La educación moral según Lawrence Kohlberg. Barcelona, Gedisa.

Martínez, M.,(1999). La psicología humanista: un nuevo paradigma psicológico. México: Trillas.

Morrison, K., Robbins, H. y Rose, D., (2008). Operationalizing culturally relevant pedagogy: A synthesis of classroom-based research. Equity y Excellence in Education, 41(4), 433-452.

Ortega, R., \& Sánchez, V. (2011). Juvenile dating and violence. In C. P. Monks \& I. Coyne (Eds.) Bullying in different contexts. (pp. 113-136). Cambridge: Cambridge University Press. 
Ortega, R.; Del Rey, R. y Fernández, I. (2003). Working together to prevent school violence: The Spanish response. En P. K. Smith (ed.), Violence in Schools: The Response in Europe (pp. 209- 240) Londres: Routledge.

Programa de Promoción de la Reforma Educativa en América Latina y el Caribe (2003). Deserción escolar: Un problema urgente que hay que abordar. Formas y Reformas de la Educación, Serie Políticas, vol. 5, n. 14, 1-4.

Ramírez, S. y Justicia, F. (2006). El maltrato entre escolares y otras conducta -problema para la convivencia. Revista Electrónica de Investigación Psicoeducativa, $9,4,265-290$.

Ray, J. (2005). Beyond tolerance and persecution. Reassesing our approach to Medieval convivencia. Jewish Social Studies, 11(2), 1-18.

Shyman, E. (2011). A comparison of the concepts of democracy and experience in a sample of major works by Dewey and Freire. Educational Philosophy and Theory, 43(10), 1035-1046.

Trianes, M.V. y Fernández-Figarés, C. (2001). Aprender a ser persona y a convivir: Un programa para secundaria. Bilbao: Descleé de Brower.

Touriñán, J. M. (2005). Educación en valores; educación intercultural y formación para la convivencia pacífica. Eduga: Revista Galega do Ensino, 13(47), 1367-1418.

Valoras UC (2017). Nuestra propuesta. http://valoras.uc.cl/quienes-somos/nuestrapropuesta

Viguer, P. y Avià, S. (2009). Un modelo local para la promoción de la convivencia y la prevención de la violencia entre iguales desde el ámbito comunitario. Cultura y Educación, 21(3), 345-359.

Vygotsky, L. (1993). Pensamiento y Lenguaje. En L. Vygotsky, Obras Escogidas (Tomo II). Madrid: Visor. (Trabajo original de 1934).

Westheimer, J. y Kahne, J. (2004). Educating the "Good" Citizen: Political Choices and Pedagogical Goals. Political Science and Politics, 37(2), 241-247. 